\title{
Immunosuppressive therapy in pancreas and islet transplant: Need for simultaneous assessment of insulin sensitivity and secretion ${ }^{*}$
}

\author{
Stefano Benedini ${ }^{1,2 \#}$, Andrea Caumo ${ }^{2}$, Ileana Terruzzi ${ }^{3}$, Livio Luzi ${ }^{1,2}$ \\ ${ }^{1}$ Research Center of Metabolism, IRCCS Policlinico San Donato Milanese, Milan, Italy; \\ \#Corresponding Author: stefano.benedini@unimi.it \\ ${ }^{2}$ Department of Biomedical and Health Sciences, University of Milan, Milan, Italy; \\ ${ }^{3}$ Division of Metabolic and Cardiovascular Sciences, San Raffaele Scientific Institute, Milan, Italy
}

Received 31 May 2013; revised 30 June 2013; accepted 8 July 2013

Copyright (C) 2013 Stefano Benedini et al. This is an open access article distributed under the Creative Commons Attribution License, which permits unrestricted use, distribution, and reproduction in any medium, provided the original work is properly cited.

\begin{abstract}
Diabetes mellitus is a metabolic disease possible to treat via pancreas/islet transplantation but most immunosuppressive drugs are diabetogenic. In this letter, we review current up to date methods to assess insulin action and secretion (using the surrogate indexes) suggesting their use in large studies in populations of pancreas/ islets transplanted patients.
\end{abstract}

Keywords: Pancreas-Islets Transplantation; Insulin Sensitivity; Insulin Secretion; HOMA (The Homeostasis Model Assessment); QUICKI (The Quantitative Insulin-Sensitivity Check Index); Hyperinsulinemic Clamp

\section{INTRODUCTION}

At present, pancreas and islet-cell transplantation are the only potential cures for type I diabetes, but long-term immunosuppression may produce several severe metabolic complications, such as glucose intolerance or overt diabetes.

It is now 25 years since the antiproliferative and immunosuppressive properties of rapamycin were first reported [1]. The latter effects were initially overshadowed by the former, and the agent was pursued more for its anticancer [2] than its potential transplantation effects. It is structurally similar to FK506, but unlike the calcineurin inhibitors, it has no effect on interleukin-2 production [3], despite clearly interfering with antigenstimulated lymphocyte responses. The discovery that in

*The authors declare no conflicts of interest that are directly relevant to the content of this article. vitro it would compete for the FK-binding protein and displace FK506 [4] gave rise to the misconception that the use of these two agents together would not be possible. Furthermore, in literature rapamycin has been demonstrated its strong potency compared to other agents.

Rapamycin is a potent inhibitor of endothelial injury in rat aortic allografts. When added to full-dose cyclosporine it achieves low rejection rates, but it augments the nephrotoxicity and hyperlipidemia of cyclosporine. On the other hand, it allows discontinuation of calcineurin inhibitors in stable kidney and liver patients suffering from nephrotoxicity late posttransplant. At least in Caucasian patients, discontinuation of cyclosporine is possible as early as 3 months post-kidney transplant. In combination with low-dose tacrolimus, exceptionally low rates of rejection were seen in recipients of kidney, pancreas, and liver recipients with preservation of excellent renal function. These pilot studies have been confirmed in several single-centre and, more recently, multicentre trials in kidney and pancreas transplantation. The side-effect profile of hyperlipidemia, lymphocoeles, delay wound healing, and possibly liver effects are coming into focus, and ways of minimizing these problems are being introduced. The lessons learned include the need for early adequate blood levels, the lack of correlation between dose and drug exposure, and the potency that allows marked dose reductions in calcineurin inhibitors and steroids [5]. The avoidance of steroidal immunosuppressive drugs dramatically reduced the derangements of glucose metabolism. This was shown by our research group in other populations of organ transplant patients, such as liver [6] and kidney recipients [7]. Moreover, cyclosporine A is known both to impair insulin secretion and to decrease insulin action [8] and the nephrotoxicity is a major problem of this therapy [9]. 


\section{METHODOLOGY}

We have recently shown that successful intraportal islet transplantation can normalize hepatic glucose production and insulin action in type 1 diabetic patients with a kidney transplant [10]. This procedure is relatively safe, noninvasive (percutaneous puncture of the liver) and repeatable, and it is performed in several centers worldwide $[11,12]$. The major factors limiting the large-scale application of islet graft in diabetic patients receiving chronic immunosuppression for a kidney graft are 1) the low percentage of patients reaching insulin independence and a complete normalization of glucose homeostasis [10]; and 2) the limited survival of fully successful grafts. Most diabetic patients receiving an islet graft achieve only partial function and a reduction of the pretransplant insulin requirement [11-16]; they are characterized by fasting C-peptide concentrations in the near-normal range, but frankly abnormal fasting glucose and glycosylated hemoglobin [11-14]. In the last decade, we [15,16] and others [17] found that protein and lipid metabolism had greater sensitivity to acutely infused insulin than did glucose metabolism, both in diabetes and uremia.

The isoglycemic, hyperinsulinemic clamp is aimed to study the effect of insulin on glucose metabolism while glycaemia is maintained at the basal, pre-test level by means of an exogenous glucose infusion, whereas the hyperglycemic hyperinsulinemic clamp is considered the gold standard for the assessment of insulin secretion (Figure 1).

The HOMA index (HOMA stands for homeostatic model assessment) measuring insulin sensitivity (HOMAIS) is calculated as follows: HOMA-IS $=22.5 /(\mathrm{gb} \times \mathrm{ib})$, where gb and ib are the basal glucose and insulin levels, respectively. The HOMA index measuring beta-cell secretion (HOMA-beta-cell) is calculated as follows: (HOMA-beta cell $=20 \mathrm{ib} /($ gb-3.5). Over the recent years the popularity of the HOMA indexes has been boosted by a number of validation studies and has been gained a large success as the most economic way to measure concurrently insulin sensitivity and secretion [18]. Among the various dynamic tests that are available to the investigator, the oral glucose tolerance test has several advantages. It is very simple, it does not require trained personnel, the discomfort for the patient is minimized, the perturbation is physiological. The results are so promising that it can be envisioned that in few years the OGTT will become the method for the simultaneous assessment of glucose tolerance, insulin secretion and insulin sensitivity. As far as insulin secretion is concerned, one should bear in mind that during the OGTT the firstand second-phase insulin secretion are not clearly distinguishable. What can be determined are two components of insulin secretion, namely the dynamic and the static components. The former component is related to the sensitivity of the beta-cell to the rate of change of glucose, while the latter component is related to the sensitivity of the beta-cell to the excursion of glucose (Figure 2).

An additional oral model (more physiological than the OGTT) is the mixed meal, which is able to asses both insulin sensitivity and beta cell function (by disposition index) like the OGTT [19].

The IVGTT consists in rapidly injecting a bolus of glucose intravenously. The IVGTT allows the investigator to clearly distinguish the first-phase insulin secretion
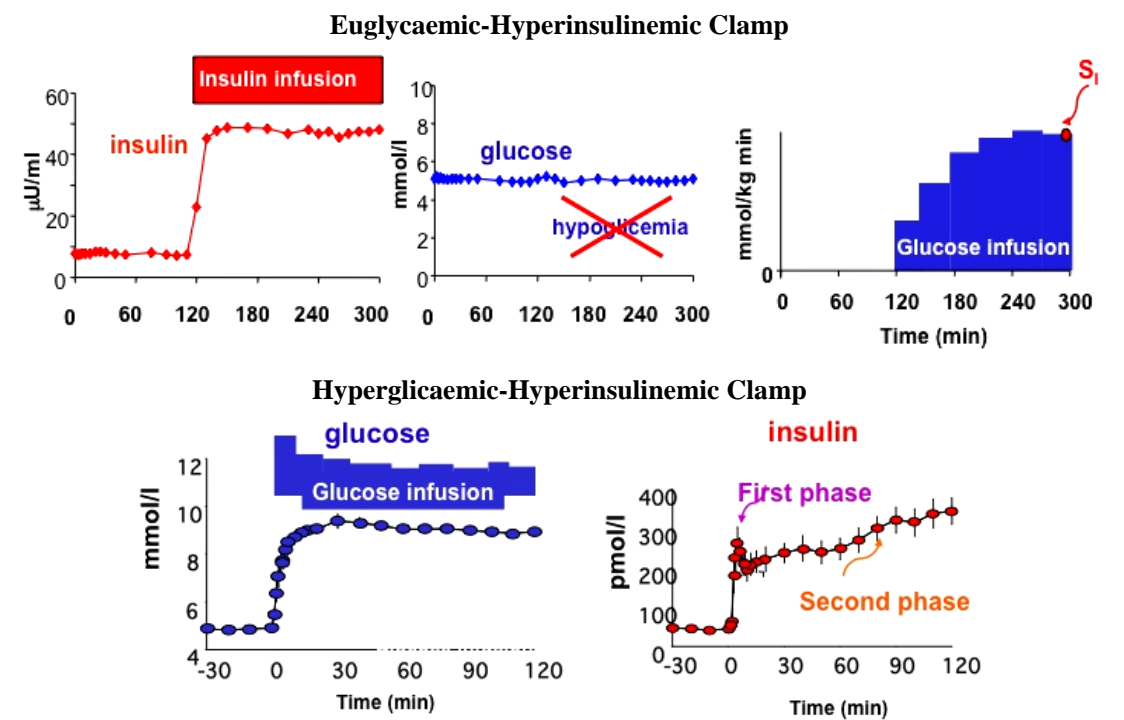

Figure 1. Schematic representation of Euglycaemic-Hyperinsulinemic Clamp (upper panel) and Hyperglicaemic-Hyperinsulinemic Clamp (lower panel) with graphic representation of glucose and insulin plasma concentrations. 


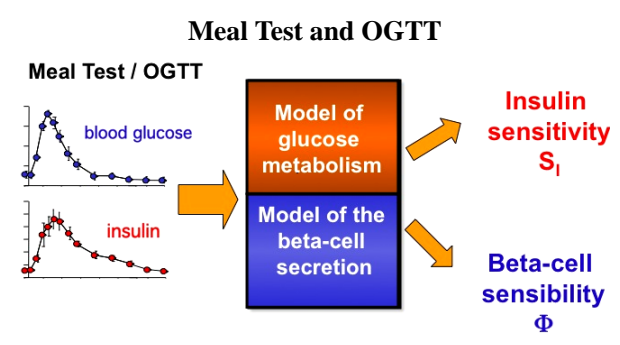

Figure 2. Meal Test and Oral Glucose Tolerance Test (OGTT) with graphic representation of glucose and insulin plasma concentrations during these tests and models for determination of insulin sensitivity and insulin action.

and to calculate the glucose tolerance of the subject from the slope of the glucose decay curve. In addition, if the glucose bolus is followed at 20 min by an insulin bolus, the insulin sensitivity of the subject under study can be determined from the minimal model analysis of the glucose and insulin data sets. The IVGTT is more laborintensive than the OGTT. The advantage of the IVGTT over the OGTT is that the first-phase of insulin response is clearly visible despite being more physiological (Figure 3).

\section{DISCUSSION}

Successful pancreas transplantation has already been shown to correct the metabolic abnormalities of IDDM [7,20]. In 1996 our work demonstrated that intraportal islet transplantation (a procedure associated with a much lower morbidity) also normalized hepatic glucose production and improved tissue glucose utilization in a selected cohort of diabetic patients [21]. Moreover, a study conducted by our group demonstrated that two indices of insulin sensitivity (HOMA-IS and QUICKI) were reliable measures of insulin sensitivity in kidney-pancreas transplanted patients [22].

A recent paper showed in a multiethnic cohort of U.S. postmenopausal women that high HOMA-IR (insulin resistance) and low HOMA-beta-cell were independently and consistently associated with an increased diabetes risk. These data suggest the value of HOMA indexes for diabetes risk in epidemiologic studies [23].

In type 2 diabetes, the loss of the early surge of insulin release, associated to insulin resistance, is an early and quite common defect that may have a pathogenetic role in the development of postprandial hyperglycemia [24]. Therefore in Pancreas and Islet transplanted patients is mandatory to have information about both insulin sensitivity and beta-cell function. In fact the early detection of graft dysfunction can facilitate the management of transplanted patients for optimizing the anti-rejection therapies. The increasing need to keep the experimental costs as low as possible has given remarkable impulse to the

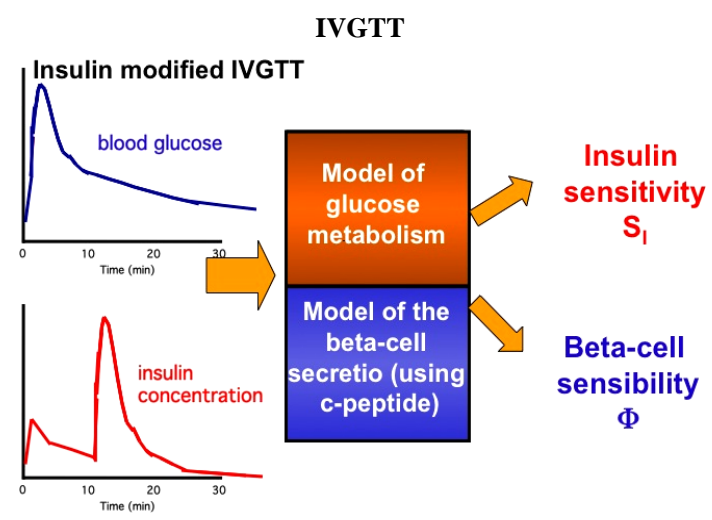

Figure 3. IntraVenous Glucose Tolerance Test (IVGTT) with graphic representation of glucose and insulin plasma concentrations during these tests and models for determination of insulin sensitivity and insulin action.

use of surrogate indexes of insulin sensitivity and secretion derived from the measurements of glycemia and insulinemia obtained from a single blood sample drawn in the postabsorptive state. The indexes that championed this trend since from the beginning are the so-called HOMA indexes. Another study performed by our group showed that HOMA-IS and QUICKI (in comparison with isoglycemic, hyperinsulinemic clamp) are simple reliable tools to assess insulin sensitivity in clinical and epidemiologic investigations of chronic liver disease before and after LTx [25].

An interesting review by Wallace and associated about the use and abuse of model for assessment of beta-cell function and insulin sensitivity showed that it is possible to use HOMA to assess insulin sensitivity in subjects treated with insulin, but it is imperative to ensure that samples are taken when glucose and insulin concentrations are in a steady state. Therefore this model does not apply when a subject is being treated with exogenous insulin. The use of HOMA in subjects on insulin needs further validation, and studies aiming at examining the use of HOMA under these circumstances are in progress [26].

If the investigator needs a more accurate quantification of insulin secretion and insulin sensitivity, dynamic tests are mandatory. The burden for the clinical investigator and the discomfort for the patient increase, nevertheless the information gathered from this type of studies is more reliable than the one provided by the HOMA-indices, especially concerning insulin secretion.

A recent paper by Cobelli and associates demonstrated that both IVGTT and meal tolerance tests could be used to illustrate how these different approaches would provide complimentary yet differing insights regarding the regulation of beta cell function in humans [27].

Most immunosuppressive drugs (if not all of them) 
have metabolic effects and impair insulin action [28], acting at different levels in the postinsulin receptor signaling cascade and the final effector metabolic pathways of carbohydrate, protein, and lipid/lipoprotein metabolism. Prednisone is well known to induce insulin resistance with respect to glucose metabolism [7]. Both cyclosporine and FK506 were shown by us to determine a modulation of insulin secretion and insulin action in liver-transplanted patients [29]. Rapamycin selectively inhibits the phosphorylation of p70 S6 kinase in vitro [30].

The preexisting disease leading to organ transplantation is important in facilitating the de novo development of diabetes mellitus. The worst theoretical condition regards patients already diabetic prior to transplantation. This is the rule for pancreas-transplanted patients and is quite common for kidney-transplanted patients who receive the organ for end-stage diabetic nephropathy.

In particular, the major metabolic advantage of the endocrine pancreas transplantation over other therapeutic modalities of treatment of Insulin-Dependent Diabetes Mellitus is that it represents a closed-loop system, with a secretory pattern elicited by glucose and other secretagogue stimuli.

The assessment of insulin secretion is of paramount importance to evaluate the success of an islet graft, to monitor the beta-cell function as time elapses, to detect the early signs of rejection. Nevertheless, since glucose tolerance depends on both insulin secretion and insulin sensitivity, the combined assessment of insulin secretion and insulin sensitivity from the same clinical test would provide the investigator a more complete picture of the metabolic status of the transplanted patient.

A different interesting consideration is that, independently from the immunosuppressive therapy, the metabolic condition and the genetic background of the donor for pancreas and islet transplantation are important determinants for the success of the transplant itself and for the probability of developing metabolic alterations after organ transplantation.

The easiest and consequently most popular assessment of beta-cell function is the homeostatic responsivity index HOMA-beta-cell. The homeostatic model HOMA-IS provides also an index of insulin sensitivity, thus permitting the evaluation of beta-cell function in relation to the prevailing insulin action [27].

In addition, the oral minimal model, using an OGTT or a mixed meal, can be used to measure beta cell function, insulin sensitivity and hepatic insulin extraction of pancreas/islet transplanted patients.

\section{CONCLUSIONS}

A combination of hyperglycemic and euglycemic-hy- perinsulinemic clamp studies supplies the gold standard for quantifying of insulin sensitivity and beta-cell function. However, clamp studies are expensive, complex to perform and require highly trained personnel [31].

The good markers for monitoring glucose metabolism in patients with pancreas and islet transplant are now disposable for all patients with simply blood basal sample. In particular HOMA-IS, QUICKI and revised QUICKI are indexes of insulin sensitivity validated in many transplanted patients (liver and kidney-pancreas transplanted). Among the various dynamic tests, the OGTT and the mixed meal (able to assess both insulin sensitivity and beta cell function) are more appropriate in this category of transplanted patients than IVGTT.

Therefore, HOMA-IS, HOMA-beta-cell, QUICKI and revised QUICKI are more simple and less invasive. In patients with pancreas and islet transplant, where glucose metabolism must be observed repeatedly during the first weeks after the allograft, these indexes were shown to be a reliable surrogate measure of in vivo insulin sensitivity/ secretion compared to the euglycaemic/hyperglycaemic clamp technique.

\section{REFERENCES}

[1] Martel, R.R, Klicius, J. and Galet, S. (1977) Inhibition of the immune response by rapamycin, a new antifungal antibiotic. Canadian Journal of Physiology and Pharmacology, 55, 48-51. doi:10.1139/y77-007

[2] Douros, J. and Suffness, M. (1981) New antitumor substances of natural origin. Cancer Treatment Reviews, 8 63-87. doi:10.1016/S0305-7372(81)80006-0

[3] Dumont, J., Staruch, M.J., Koprak, S.L., et al. (1992) The immunosuppressive and toxic effects of FK-506 are mechanistically related: Pharmacology of a novel antagonist of FK-506 and rapamycinI. The Journal of Experimental Medicine, 176, 51.

[4] Liu, J., Farmer, J.D., Lane, W.S., et al. (1991) Calcineurin is a common target of cyclophilin-cyclosporin A and FKBP-FK506 complexes. Cell, 66, 807-815. doi:10.1016/0092-8674(91)90124-H

[5] MacDonald, A.S. (2003) Rapamycin in combination with cyclosporine or tacrolimus in liver, pancreas, and kidney transplantation. Transplantation Proceedings, 35, 201S208S. doi:10.1016/S0041-1345(03)00231-8

[6] Perseghin, G., Regalia, E., Battezzati, A., et al. (1997) Regulation of glucose homeostasis in humans with denervated livers. Journal of Clinical Investigation, 100, 931-941. doi:10.1172/JCI119609

[7] Luzi, L., Secchi, A., Facchini, F., et al. (1990) Reduction of insulin resistance by combined kidney-pancreas transplantation in type 1 (insulin-dependent) diabetic patients. Diabetologia, 33, 549-556. doi:10.1007/BF00404143

[8] Gillkinson, S.G., Bartlett, S.T. and Cury, D.L. (1989) Synthesis-secretion coupling of insulin: Effect of cyclosporin. Diabetes, 38, 464-470. 
[9] Shapiro, R. (2004) Low toxicity immunosuppressive protocols in renal transplantation. The Keio Journal of Medicine, 53, 18-22. doi:10.2302/kjm.53.18

[10] Luzi, L., Perseghin, G., Brendel, M.D., et al. (2001) Metabolic effects of restoring partial beta-cell function after islet allotransplantation in type 1 diabetic patients. Diabetes, 50, 277-282. doi:10.2337/diabetes.50.2.277

[11] Bretzel, R.G., Browatzki, C.C., Schultz, A., et al. (1993) Clinical islet transplantation in diabetes mellitus (German). Diabetes Stoffwechsel und Herz, 2, 378-390.

[12] Alejandro, R., Mintz, D.H., Noel, J., et al. (1987) Islet cell transplantation in type 1 diabetes mellitus. Transplantation Proceedings, 19, 2359-2361.

[13] London, N.J., Robertson, G.S., Chadwick, D.R., et al. (1994) Human pancreatic islet isolation and transplantation. Clinical Transplantation, 8, 421-459.

[14] Luzi, L., Castellino, P., Simonson, D.C., et al. (1990) Leucine metabolism in insulin-dependent diabetes mellitus: Role of insulin and substrate availability. Diabetes, 39, 38-48. doi:10.2337/diabetes.39.1.38

[15] Luzi, L., Petrides, A.S. and DeFronzo, R.A. (1993) Different sensitivity to insulin of glucose and amino acid metabolism in NIDDM. Diabetes, 42, 1868-1877. doi:10.2337/diabetes.42.12.1868

[16] Luzi, L, Groop, L.C., Perseghin, G, et al. (1996) Effect of pancreas transplantation on free fatty acids metabolism in uremic IDDM patients. Diabetes, 45, 354 -360. doi:10.2337/diabetes.45.3.354

[17] Groop, L.C., Bonadonna, R.C., Del Prato, S., et al. (1989) Glucose and free fatty acids metabolism in non-insulindependent diabetes mellitus: Evidence for multiple sites of insulin resistance. Journal of Clinical Investigation, 84, 205-213. doi:10.1172/JCI114142

[18] Matthews, D.R., Hosker, J.P., Rudenski, A.S., Naylor, B.A., Treacher, D.F. and Turner, R.C. (1985) Homeostasis model assessment: Insulin resistance and $\beta$-cell function from fasting plasma glucose and insulin concentrations in man. Diabetologia, 28, 412-419. doi:10.1007/BF00280883

[19] Dallla, M.C, Caumo, A., Basu, R., Rizza, R., Toffolo, G.M. and Cobelli, C. (2004) Minimal model estimation of glucose absorption and insulin sensitivity from oral test: Validation with a tracer method. American Journal of Physiology_Endocrinology and Metabolism, 287, E637E643. doi:10.1152/ajpendo.00319.2003

[20] Perseghin, G., Hilden, H., Bianchi, E., et al. (1993) Normal sensitivity of FFA metabolism despite resistance of glucose metabolism to insulin after pancreas transplantation. Diabetes, 43, 138a.

[21] Luzi, L., Hering, B.J., Socci, C., et al. (1996) Metabolic effects of successful intraportal islet transplantation in insulin-dependent diabetes mellitus. Journal of Clinical In- vestigation, 97, 2611-2618. doi:10.1172/JCI118710

[22] Perseghin, G., Caumo, A, Sereni, L.P., et al. (2002) Fasting blood sample-based assessment of insulin sensitivity in kidney-pancreas-transplanted patients. Diabetes Care, 25, 2207-2211. doi:10.2337/diacare.25.12.2207

[23] Song, Y., Manson, J.E., Tinker, L., Howard, B.V., Kuller, L.H., Nathan, L., Rifai, N. and Liu, S. (2007) Insulin sensitivity and insulin secretion determined by homeostasis model assessment and risk of diabetes in a multiethnic cohort of women: The Women's Health Initiative Observational Study. Diabetes Care, 30, 1747-1752. doi:10.2337/dc07-0358

[24] Del Prato, S., Marchetto, P. and Bonadonna, R.C. (2002) Phasic insulin release and metabolic regulation in type 2 diabetes. Diabetes, 51, S109-S116. doi:10.2337/diabetes.51.2007.S109

[25] Perseghin, G., Caumo, A., Mazzaferro, V., Pulvirenti, A., Piceni, S.L., Romito, R., et al. (2003) Assessment of insulin sensitivity based on a fasting blood sample in men with liver cirrhosis before and after liver transplantation. Transplantation, 76, 697-702. doi:10.1097/01.TP.0000079252.94857.8D

[26] Wallace, T.M., Levy, J.C. and Mattehews, D.R. (2004) Use and abuse of HOMA modeling. Diabetes Care, 27, 1487-1495. doi:10.2337/diacare.27.6.1487

[27] Cobelli, C., Toffolo, G.M., Dallla, M.C., Campioni, M., Denti, P., Caumo, A., Butler, P.C. and Rizza, R.A. (2007) Assessment of beta cell function in humans, simultaneously with insulin sensitivity and hepatic extraction, from intravenous and oral glucose test. American Journal of Physiology-Endocrinology and Metabolism, 293, E1E15. doi:10.1152/ajpendo.00421.2006

[28] Cai, W., Hu, L. and Foulkes, J.G. (1996) Transcriptionmodulating drugs: Mechanism and selectivity. Current Opinion in Biotechnology, 7, 608-615. doi:10.1016/S0958-1669(96)80071-1

[29] Fernandez, L.A., Lehmann, R., Luzi, L., et al. (1999) The effects of maintenance doses of FK506 versus cyclosporin A on glucose and lipid metabolism after orthotopic liver transplantation. Transplantation, 68, 1532-1541. doi:10.1097/00007890-199911270-00017

[30] Patti, M.E., Brambilla, E., Luzi, L., et al. (1998) Bidirectional modulation of insulin action by amino acids. Journal of Clinical Investigation, 101, 1519-1529. doi:10.1172/JCI1326

[31] Uwaifo, G.I., Fallon, E.M., Chin, J., Elberg, J., Parikh, S.J. and Yanovski, J.A. (2002) Indices of insulin action, disposal, and secretion derived from fasting samples and clamps in normal glucose-tolerant black and white children. Diabetes Care, 25, 2081-2087. doi:10.2337/diacare.25.11.2081 